\title{
Characterization of longitudinal impedances in storage rings via multibunch effects
}

\author{
D. Teytelman, J. Fox, and S. Prabhakar \\ Stanford Linear Accelerator Center, Stanford University, Stanford, California 94309 \\ J. M. Byrd \\ Lawrence Berkeley National Laboratory, One Cyclotron Road, Berkeley, California 94720 \\ Department of Physics, University of California, Davis, Davis, California 95616
}

(Received 17 May 2001; published 28 November 2001)

\begin{abstract}
In this paper we present two new techniques for frequency-resolved characterization of longitudinal impedances in storage rings. The first method is based on transient measurements of the growth rates and tune shifts of unstable coupled-bunch modes. In the second approach, estimates of the impedances are obtained from analysis of the steady-state synchronous phases of the bunches for uneven fill patterns. These techniques are applicable to measurements of both fundamental and higher-order mode (HOM) impedances and allow characterization of shunt impedances and quality factors of the HOMs. Methods presented here are complementary to laboratory bench measurements of rf cavities, in that the beambased measurements directly sense the physical impedance in the installed configuration. Experimental results from the Advanced Light Source and BESSY-II are presented showing the use of these techniques to measure complex impedances.
\end{abstract}

DOI: 10.1103/PhysRevSTAB.4.112801

PACS numbers: 29.27.Bd, 29.20.Dh

\section{INTRODUCTION}

The interaction of charged particles in a storage ring or circular accelerator with the ring impedance determines many important accelerator dynamics parameters. Single and multibunch instabilities are the result of interactions of the bunches with the impedance of the machine, and achieving high stored currents requires knowledge and control of the ring components which produce the dominant narrow band impedances. Traditionally, longitudinal impedances in an accelerator have been studied using bench measurements of the accelerator components as well as beam-based measurements. There are several laboratory techniques to measure impedances of physical components based on propagating pulses on wires through structures, and on measurements of transfer functions of structures as ferrite beads or conducting needles are moved within a cavity volume [1,2]. Beam-based impedance measurement techniques exist as well. The integrated longitudinal impedance of a circular machine can be measured via the shift in synchronous phase versus current of a single test bunch $[3,4]$. By varying bunch length in such measurements one can estimate the parameters of the broad band equivalent impedance [5,6]. However, such techniques do not resolve individual higher-order modes (HOMs). Information about these modes is very important for predicting the thresholds of collective instabilities and for configuring accelerator and feedback systems to stabilize the beam. Beam-based techniques are an important adjunct to numerical calculations and laboratory bench measurements of rf cavities and components, in that they can measure the actual installed impedance, which is strongly influenced by coupling ports, parasitic components, and environmen- tal factors which can be difficult to include in simulations or laboratory tests.

Frequency-resolved information about the impedance can be extracted from a measurement of the beam transfer function (BTF) $[7,8]$. However, such a measurement can be performed only below the instability threshold. In addition, network analyzer sweeps have to be repeated for each unstable mode making BTF approach slow and cumbersome for machines with large numbers of coupledbunch modes. Yet another method for characterizing the impedance is through observation of cavity coupling probe signals excited by the beam [9]. This approach allows determination of the center frequencies and quality factors of resonant modes, but the coupling of these resonances to the beam is measured only qualitatively. That is, resonances that do not couple to the beam will not be excited; however, effect of the excited resonances on the beam is not clear from such a measurement. In addition, certain resonances within the rf cavity may be weakly coupled to the probe.

This paper presents several beam-based longitudinal impedance measurement techniques. These multibunch techniques measure the effective longitudinal impedance as a function of frequency in a sampling bandwidth up to the rf frequency. This effective impedance represents a sum of physical impedance components in the frequency bands $N f_{\text {rf }}$ to $(N+1) f_{\text {rf }}$ shifted to de to $f_{\mathrm{rf}}$ band and summed (the aliasing process). By comparing the effective (aliased) impedance to the bench measurements of the accelerator components various higher-order mode resonators can be identified and their complex impedance (and parameters such as center frequency and $Q$ ) measured. 
In Sec. II we present theoretical background and describe how impedances can be estimated based on coupledbunch instability measurements. The relation between impedances and synchronous phase transients is explored in Sec. III. The experimental results are presented in Sec. IV. Advantages and disadvantages of the proposed techniques are summarized in Sec. V.

\section{LONGITUDINAL IMPEDANCES AND COUPLED-BUNCH INSTABILITIES MEASURED FROM THE GROWTH OR DECAY OF COUPLED-BUNCH MODE OSCILLATION}

Bunches of charged particles passing through the vacuum chamber of a storage ring leave behind electromagnetic fields. These fields (wake fields) affect the energy of the following bunches providing a bunch-to-bunch coupling mechanism. At high beam currents such coupling can cause instabilities. The theory of coupled-bunch instabilities is well developed $[10,11]$. Here we will use it to link the characteristics of the unstable longitudinal motion to the Fourier transform of the wake function known as impedance.

The longitudinal wake function $W^{\|}(t)$ is defined as the integrated longitudinal component of the electric field experienced by a test charge passing through the vacuum chamber a time $t$ later than the particle that excites the field. The field is integrated over an entire turn. Longitudinal impedance $Z^{\|}(\omega)$ is defined as

$$
Z^{\|}(\omega)=\int_{-\infty}^{\infty} W^{\|}(t) e^{-i \omega t} d t=\int_{0}^{\infty} W^{\|}(t) e^{-i \omega t} d t .
$$

The impedance is Hermitian; that is, $Z(-\omega)=Z^{*}(\omega)$ where * denotes complex conjugate as it is a Fourier transform of a real function.

The bunch motion in a storage ring can be projected onto the orthonormal basis of the even fill eigenmodes (EFEMs). For a symmetrically filled ring with $N$ bunches, the motion of bunch $k$ oscillating in mode $l$ is given by

$$
\phi_{k}(t)=A e^{j 2 \pi k l / N} e^{\Lambda_{l} t},
$$

where $\phi_{k}$ is the phase deviation from synchronous phase, $N$ is the number of filled rf buckets, and $\Lambda_{l}$ is the modal eigenvalue [12]. If there is no longitudinal impedance affecting mode $l$ its unperturbed eigenvalue is given by

$$
\Lambda^{0}=-d_{r}+j \omega_{s},
$$

where $d_{r}$ is the radiation damping rate and $\omega_{s}$ is the synchrotron frequency. When longitudinal impedances are introduced they result in eigenvalue shift $\lambda_{l}$,

$$
\lambda_{l}=\frac{\pi \alpha e f_{\mathrm{rf}}^{2} I_{0}}{E_{0} h \omega_{s}} Z^{\| \mathrm{eff}}\left(l \omega_{0}+\omega_{s}\right),
$$

$$
Z^{\| \mathrm{eff}}(\omega)=\frac{1}{2 \pi f_{\mathrm{rf}}} \sum_{p=-\infty}^{\infty}\left(p N \omega_{0}+\omega\right) Z^{\|}\left(p N \omega_{0}+\omega\right)
$$

where $\alpha$ is the momentum compaction factor, $e$ is the charge of the electron, $f_{\mathrm{rf}}$ is the frequency in the accelerating cavities, $I_{0}$ is the beam current, $E_{0}$ is the beam energy, $h$ is the ring harmonic number, $\omega_{0}$ is the revolution frequency, and $Z^{\|}(\omega)$ is the total longitudinal impedance. Here we assume that the bunch length is much smaller than the wavelengths of the wake fields driving coupled-bunch instabilities.

Summation over $p$ in Eq. (3) describes aliasing of the longitudinal impedance into the frequency range from dc to $f_{\text {rf }}$. The aliased impedance $Z^{\text {lleff }}$ sampled at the upper synchrotron sideband of the $l$ th revolution harmonic defines the modal eigenvalue shift $\lambda_{l}$.

In order to measure modal eigenvalues $\Lambda$ we use the capabilities of a programmable longitudinal feedback system [13]. In this system the longitudinal coordinates of all bunches are measured relative to a reference oscillator. These coordinates are sampled and can be recorded in the digital memory for tens of thousands of turns. The individual measurement of the instantaneous phase of each bunch, in conjunction with the long time record of motion, is a very powerful source of information about the beam dynamics and machine impedances. The frequency resolution available in these long recordings allows measurement of coherent tunes with resolution of a few $\mathrm{Hz}$, while sampling all revolution harmonics over the full rf bandwidth. In a transient grow/damp measurement, the feedback loop is opened under software control for a predetermined period of time and then closed. In the open-loop conditions, unstable modes grow exponentially due to noise, and a feedback system records the motion of the bunches during the transient. The motion is then projected on the EFEM basis, and modal exponential growth and damping rates, as well as oscillation frequencies, are extracted [14]. Stable eigenmodes can be characterized by driving the mode of interest to a measurable oscillation level before the transient.

Once the eigenvalues of the unstable modes $\Lambda$ are measured via a grow/damp transient it is possible to extract the aliased impedance versus frequency according to Eq. (2). The aliased beam-derived impedance, combined with knowledge about the impedances from bench measurements of ring components, may then be properly assigned as an unaliased impedance versus frequency.

For this paper we will consider only resonant modes in cavitylike structures. Longitudinal impedance of such modes can be expressed as

$$
Z^{\|}(w)=\frac{R_{s}}{1+j Q\left[\omega / \omega_{r}-\omega_{r} / \omega\right]},
$$

where $\omega_{r}$ is the resonance center frequency and $R_{s}$ is the shunt impedance. The quality factor $Q$ defines the 
width of the resonance. Traditionally, resonators are considered narrow band if $Q \gg 1$ and wideband if $Q \approx 1$. In this context we will define another narrow band/wideband resonator grouping based on the width relative to the ring revolution frequency $\omega_{0}$. We will define a narrow band impedance as one exciting unstable motion of only one or two EFEMs. Conversely, wideband impedances drive multiple modes. Some of the techniques described here are best suited to the measurement of the narrow band impedances while other methods are better for the wideband cases.

Narrow band resonances are sampled by these eigenvalue measurements at a low number of points spaced by $\omega_{0}$. Thus it is necessary to move the resonant center frequency with finer resolution to fully resolve and measure the impedance parameters.

\section{LONGITUDINAL WAKE POTENTIAL AND IMPEDANCE FROM MEASURED SYNCHRONOUS PHASE TRANSIENTS}

For the cases when ring fill pattern is uneven, additional information about the impedance can be obtained from analyzing the dependence of synchronous phases on bunch currents. Previous work by Prabhakar [15] presents the relationship between the bunch currents, impedances, and synchronous phases. This work is applicable to fill patterns where all buckets are populated, however unevenly. For empty buckets synchronous phase is not measurable. Theoretically, one can define the steady-state synchronous phase for empty buckets, for example, as that of a test particle of infinitesimally small charge. However, physical measurement of that sort is infeasible. Empty buckets (gaps) are present in most uneven fill patterns, and here we will extend the analysis of synchronous phase transients to such fills.

Synchronous phase variations are caused by beam excitation of the longitudinal impedances at the revolution harmonics. The effect is illustrated in Fig. 1 showing four bunches with bunch 0 having much larger charge than others. Bunch 0 excites an oscillatory wake field. The synchronous phases of the following bunches shift by the amount proportional to the wake potential sampled at times $t=k T_{\text {rf }}$ (for small shifts). Let us define $i_{k}$ and $\phi_{k}, k=0,1, \ldots, N-1$ as vectors of bunch currents and steady-state synchronous phases, respectively. Then according to [15] we have

$$
\begin{gathered}
\Phi_{n}=\frac{-N}{\left|V_{c} \cos \left(\phi_{s}^{0}\right)\right|} I_{n} Z_{n}^{\dagger}, \\
Z_{n}^{\dagger}=\sum_{m=-\infty}^{\infty} Z^{\|}\left[(m N+n) \omega_{0}\right],
\end{gathered}
$$

where $\Phi_{n}$ and $I_{n}$ are discrete Fourier transforms (DFTs) of $\phi_{k}$ and $i_{k}$, respectively, $V_{c}$ is the peak rf cavity voltage, and $\phi_{s}^{0}$ is the synchronous phase in absence of wake fields. Summation in Eq. (6) is analogous to that in Eq. (3) and

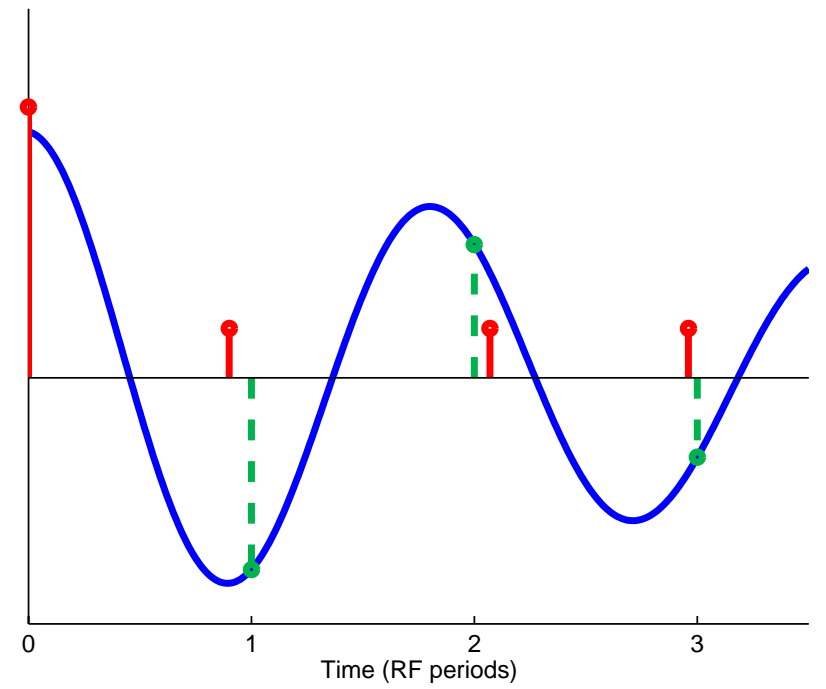

FIG. 1. (Color) Effect of the wake field on the synchronous positions of the bunches. The sketch shows the synchronous positions (spaced by $T_{\text {rf }}$ ) by green dashed lines, while the bunch arrivals are indicated by the red stems. The height of these stems is proportional to bunch charge. A large bunch (at time 0 ) produces a decaying sinusoidal wake field. The equilibrium positions of the following small bunches (near times 1,2, and 3) are altered according to the wake field sampled by the bunches.

describes aliasing of the longitudinal impedance into the 0 to $\omega_{\text {rf }}$ band. However, in this case, unlike Eq. (3), longitudinal impedance is not scaled by the frequency since in this case we are considering the equilibrium mode.

Let us define set $U$ as follows: $m \in U$ if and only if $i_{m} \neq 0$ (set of $\mathrm{rf}$ bucket numbers with nonzero stored charge). In the following discussion we will assume that a measurement of $\phi_{m}$ is available for all $m \in U$. By definition of inverse DFT,

$$
\phi_{k}=\frac{1}{N} \sum_{n=0}^{h-1} \Phi_{n} e^{j 2 \pi(n / N) k}
$$

Let us define $N \times N$ matrix $\mathbf{T}^{-1}$ as follows:

$$
\begin{aligned}
& T_{k n}^{-1}=\frac{1}{N} e^{j 2 \pi(n-1 / N)(k-1)}, \\
& \quad n=1, \ldots, N, k=1, \ldots, N .
\end{aligned}
$$

Then Eq. (7) can be written as $\vec{\phi}=\mathbf{T}^{-1} \vec{\Phi}$. Combining this with Eq. (5) we get

$$
\begin{gathered}
\vec{\phi}=\frac{-N}{\left|V_{c} \cos \left(\phi_{s}^{0}\right)\right|} \mathbf{A} \vec{Z}^{\dagger} \\
\mathbf{A}=\mathbf{T}^{-1} \operatorname{diag}(\vec{I})
\end{gathered}
$$

Equation (8) describes an $N \times N$ linear system of equations with complex coefficients and unknowns. Since $\phi$ is measured only for $m \in U$, equations described by rows of matrix A with indexes outside set $U$ are not defined. In addition, it is evident from Eq. (9) that elements of $\vec{Z}^{\dagger}$ corresponding to weakly excited components of $\vec{I}$ are not well defined. We will choose threshold level $I_{\text {thresh }}$ to 
separate large components of $\vec{I}$. Selection of the threshold is dependent on the signal-to-noise ratio in the measurement of bunch currents and synchronous phases as well as the values of $N, V_{c}$, and $\phi_{s}^{0}$. Let us define set $V$ such that $k \in V$ if and only if $\left|I_{k}\right|>I_{\text {thresh (set of revolution har- }}$ monic numbers with sufficient excitation). Defining matrix $\mathbf{A}^{U V}$ as consisting of rows $U$ and columns $V$ of matrix $\mathbf{A}$ we can write

$$
\vec{\phi}_{U}=\frac{-N}{\left|V_{c} \cos \left(\phi_{s}^{0}\right)\right|} \mathbf{A}^{U V} \vec{Z}_{V}^{\dagger} .
$$

In the DFT of bunch currents, dc term $\left(I_{0}\right)$ is the largest, so it is always included in $V$. Also, as mentioned in Sec. II, impedance is Hermitian, so that $Z_{k}^{\dagger}=Z_{N-k}^{\dagger *}$. Equation (10) can be rewritten as a real linear system of $M_{U}$ equations with $M_{V}$ unknowns, where $M_{U}$ and $M_{V}$ are the sizes of sets $U$ and $V$, respectively. Since only a few frequencies are strongly excited by the fill pattern we normally have $M_{V} \ll M_{U}$. Thus we have an overdetermined system of equations. Solving that system in the leastsquares sense we obtain $\vec{Z}_{V}^{\dagger}$.

\section{EXPERIMENTAL RESULTS}

We will illustrate impedance characterization techniques described above with measurements performed at the Advanced Light Source (ALS) and BESSY-II. Main parameters for both accelerators are summarized in Table I.

\section{A. ALS measurements}

The goal of the first measurement is to quantify the HOM impedances of the two $500 \mathrm{MHz}$ main rf cavities installed at the ALS. Past longitudinal instability measurements have determined that there are two dominant EFEMs, modes 205 and 233, excited by the impedances in the main rf cavities [16]. Using the laboratory bench measurements made on the spare cavity identical to the ones installed in the ring, mode 205 had been identified as driven by the $\mathrm{TM}_{011}$ longitudinal mode at $812 \mathrm{MHz}$. Mode 233 has two potential driving HOMs, at 2.353 and $2.853 \mathrm{GHz}$ [17]. The aliased effective impedance for ALS, as determined from the bench measurements, is illustrated in Fig. 2. The three above-mentioned resonances in the main rf cavities are marked as well as a strong HOM at $2324 \mathrm{MHz}$ in the bunch-lengthening third harmonic cavities.

Because of technical limitations it is possible to fill only $320 \mathrm{rf}$ buckets at the ALS. All of the transient measurements described here were taken with 320 equally filled buckets followed by a gap of eight rf buckets. Since the gap is small the eigenmodes of the fill are close to those of an even fill. ${ }^{1}$ A group of instability measurements was made in order to quantify the measurement noise. From 17 independent growth transients at $80 \mathrm{~mA}$ total beam current we find $\lambda_{233}=(0.43 \pm 0.02)-(3.36 \pm 0.02) j \mathrm{~ms}^{-1}$. Both the real and the imaginary parts of eigenvalue shift have very small standard deviations. The errors are due to the measurement noise and the variations in operating conditions of the accelerator that cause changes in the growth rates.

In order to characterize the frequency dependence of the impedance we shifted the center frequencies of the cavity HOM resonances by changing the temperature of the cavity. In these measurements we swept the cavity temperature set point in 11 steps over a range of $15^{\circ} \mathrm{C}$. At each point the temperature was allowed to stabilize and the open-loop eigenvalues of the unstable modes were measured using the transient grow/damp technique. During the sweep of each cavity the temperature of the other cavity was held constant. In Fig. 3 the growth rates and oscillation frequencies of modes 205 and 233 are plotted versus temperature of cavity 2 . As the total beam current changes during the experiment, all of the measurements were scaled to a reference current $I_{\text {ref }}(100 \mathrm{~mA})$ as follows:

$$
\begin{aligned}
& \lambda_{m}^{*}=\left(\lambda_{m}+d_{r}\right) \frac{I_{\mathrm{ref}}}{I_{m}}-d_{r}, \\
& \omega_{m}^{*}=\left(\omega_{m}-\omega_{s}\right) \frac{I_{\mathrm{ref}}}{I_{m}}+\omega_{s},
\end{aligned}
$$

\footnotetext{
${ }^{1}$ According to Eqs. 7.10-7.13 in [12], diagonal coupling matrix of an even fill is modified in this case with off-diagonal entries that are $8 / 320=1 / 40$ of the magnitude of the on-diagonal entries. The resulting difference between the eigenvalues of the uneven and even fills is negligible.
}

TABLE I. Nominal ALS and BESSY-II parameters.

\begin{tabular}{clcc}
\hline \hline Parameter & \multicolumn{1}{c}{ Description } & ALS & BESSY-II \\
\hline$E$ & Beam energy $(\mathrm{GeV})$ & 1.5 & 1.7 \\
$C$ & Circumference $(\mathrm{m})$ & 196.8 & 240 \\
$f_{\text {rf }}$ & rf frequency $(\mathrm{MHz})$ & 499.660 & 499.654 \\
$h$ & Harmonic number & 328 & 400 \\
$f_{\text {rev }}$ & Rotation frequency $(\mathrm{MHz})$ & 1.5233 & 1.25 \\
$\alpha$ & Momentum compaction & $1.62 \times 10^{-3}$ & $7.8 \times 10^{-4}$ \\
$U_{0}$ & Radiation loss/turn (keV) & 94 & 170 \\
$V_{\mathrm{rf}}$ & Main rf voltage $(\mathrm{MV})$ & 1.15 & 1.29 \\
$f_{s}$ & Synchrotron frequency $(\mathrm{kHz})$ & 12.25 & 7.4 \\
\hline \hline
\end{tabular}




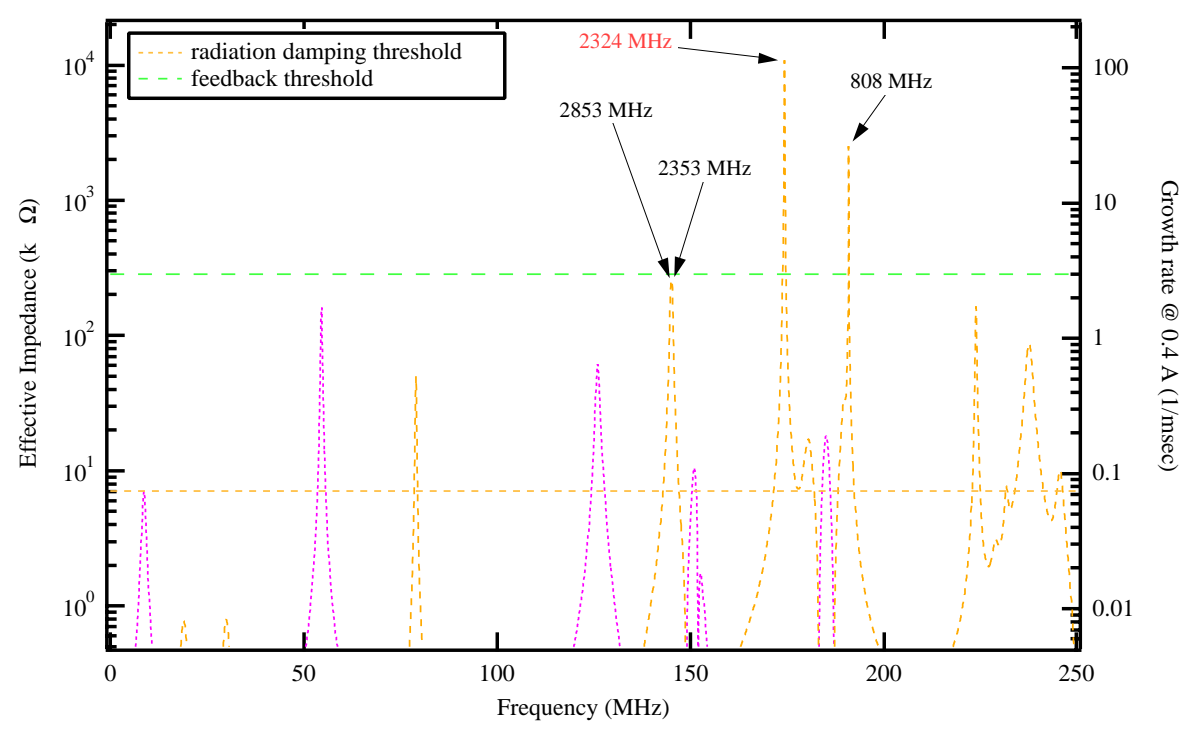

FIG. 2. (Color) Real part of the aliased longitudinal impedance obtained from the laboratory bench measurements. $Z^{\text {lleff }}$ between 250 and $500 \mathrm{MHz}$ is folded into the $0-250 \mathrm{MHz}$ range and shown in yellow.

where $\lambda_{m}$ is the measured growth rate, $\omega_{m}$ is the measured oscillation frequency, and $I_{m}$ is the beam current during the transient measurement.

The expected effect of changes in cavity temperature is to change the center frequency $\omega_{r}$ of the narrow band HOMs $[18,19]$. Since the relative change in frequency is small, the relationship between cavity temperature and
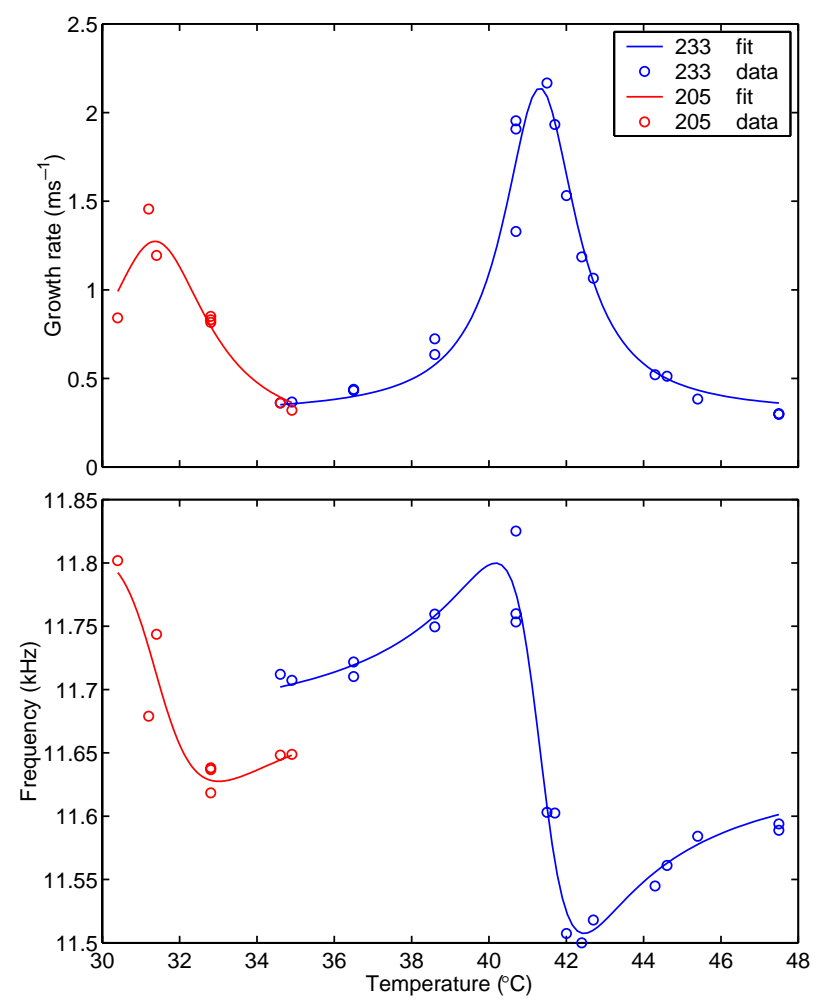

FIG. 3. (Color) Growth rates (top) and oscillation frequencies (bottom) of modes 205 and 233 in main rf cavity 2 normalized to $I_{\text {ref }}=100 \mathrm{~mA}$. center frequency is linear of the form

$$
\omega_{r}(T)=\omega_{r}\left(T_{0}\right)+K\left(T-T_{0}\right),
$$

where $T_{0}$ is the arbitrary reference temperature and $K$ is the temperature to frequency conversion gain. The measurements presented above agree well with this model. However, these measurements do not provide a means to distinguish between the two possible HOMs at 2.353 and $2.853 \mathrm{GHz}$ as the source of the aliased impedance. To resolve this ambiguity, the ring was filled with a single bunch while a cavity probe signal was monitored on a spectrum analyzer. We observed that change of cavity temperature had a very small effect on the magnitude of the revolution harmonics excited within the $2.353 \mathrm{GHz}$ resonance while the amplitude of the probe signal at $2.853 \mathrm{GHz}$ revolution harmonic scaled with temperature in agreement with the growth rate measurements. This leads to two conclusions. First, the resonance measured in the temperature scan is at $2.853 \mathrm{GHz}$. Second, we can consider the impedance presented by the $2.353 \mathrm{GHz} \mathrm{HOM}$ as constant when the temperature is changed.

Once the HOM has been identified we can determine the shunt impedance $R_{s}$ from the $\lambda_{m}^{*}$. However, in order to quantify the quality factor $Q$ we need to determine the dependence of the resonator center frequency $\omega_{r}$ on the cavity temperature. We will use the relationship presented in Eq. (11) and select the reference temperature $T_{0}$ to correspond to the center frequency of the HOM at the upper synchrotron sideband of the 233rd revolution harmonic above $5 \omega_{\text {rf }}$ (critical temperature as defined in [18]). In this case the induced growth rate is largest at $T_{0}$. Equation (11) becomes

$$
\begin{aligned}
\omega_{r} & =\omega_{233}+\omega_{s}+K\left(T-T_{0}\right), \\
\omega_{233} & =5 \omega_{\mathrm{rf}}+233 \omega_{0} .
\end{aligned}
$$


In order to measure $K$, a single bunch in the ring was filled to a nominal per bunch charge and the cavity probe signal at $\omega_{233}$ was measured at two temperatures, $T_{1}$ and $T_{2}$. At the cavity temperature $T_{1}$ the ring $\mathrm{rf}$ frequency was increased by $\Delta \omega$ so that the probe signal magnitude equaled that measured at $T_{2}$. The temperature to frequency conversion gain $K$ can then be computed as

$$
K=\frac{\omega_{233} \Delta \omega}{\omega_{\mathrm{rf}}\left(T_{2}-T_{1}\right)} .
$$

Let us consider the eigenvalue of mode $233, \Lambda_{233}$. From Eqs. (1)-(3),

$$
\begin{aligned}
\Lambda_{233}= & \Lambda^{0}+\lambda_{233} \\
= & -d_{r}+j \omega_{s}+\lambda_{\mathrm{ext}} \\
& +\frac{\pi \alpha e f_{\mathrm{rf}}^{2} I_{0}}{E_{0} h \omega_{s}} Z_{2.853 \mathrm{GHz}}^{\| \mathrm{eff}}\left(233 \omega_{0}+\omega_{s}\right), \\
Z_{2.853 \mathrm{GHz}}^{\| \mathrm{eff}}(\omega)= & \frac{5 \omega_{\mathrm{rf}}+\omega}{\omega_{\mathrm{rf}}} Z_{2.853 \mathrm{GHz}}^{\|}\left(5 \omega_{\mathrm{rf}}+\omega\right), \\
Z_{2.853 \mathrm{GHz}}^{\|}(\omega)= & \frac{R_{s}}{\left.1+i Q\left[\omega / \omega_{r}-\omega_{r} / \omega\right]\right)},
\end{aligned}
$$

where $\lambda_{\text {ext }}$ is mode 233 eigenvalue shift due to impedances other than $2.853 \mathrm{GHz}$ resonance in the cavity of interest. We can parametrize $\Lambda_{233}$ as a function of five real variables: $R_{s}, Q, T_{0}, \operatorname{Re}\left(\lambda_{\text {ext }}\right)$, and $\operatorname{Im}\left(\lambda_{\text {ext }}\right)$. Using a nonlinear least-squares estimation we extract parameter values. Figure 4 shows the measured and fitted growth rates and oscillation frequencies for the main rf cavity 1 .

Additionally, we compared the impedance parameters with the cavity probe measurements taken with a single bunch fill. The signal level at $\omega_{233}$ and neighboring revolution harmonics excited by the beam are measured with the spectrum analyzer. These measurements spanning three different temperatures were normalized for current and scaled to $T_{0}$ using the measured temperature to frequency coefficient $K$. In Fig. 5 the cavity probe signal is shown to have good agreement with the magnitude of the impedance extracted from the transient measurements.

In Table II results for both cavities are summarized. Data from numerical computations and bench measurements of a spare rf cavity are included $[2,20]$. Both numerical modeling and bench measurements have large errors in their estimates of the shunt impedances. ${ }^{2}$ As for the center frequency, the error of bench-based measurement is quite small, only $600 \mathrm{kHz}$. However, this degree of accuracy is insufficient for prediction of the coupled-bunch instability growth rates as well as determination of the optimal cavity operating temperatures. Note that characteristics of the

\footnotetext{
${ }^{2}$ In the bench measurement, the shunt impedance is computed by multiplying the measured $Q$ value by the $R / Q$ calculated using the URMEL-T computer code.
}

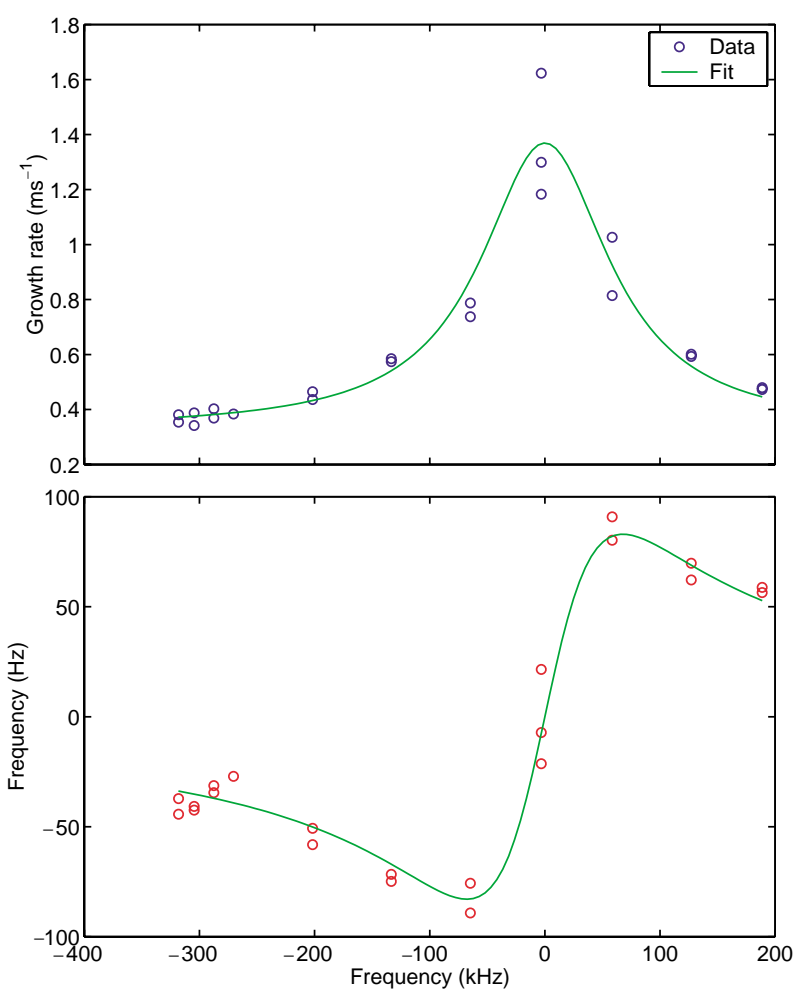

FIG. 4. (Color) Least-squares fit to the measured growth rates (top) and the measured coherent frequency shifts (bottom) versus resonator center frequency for cavity 1 .

$2.853 \mathrm{GHz}$ resonances in two cavities differ significantly. The cavities have if windows of different designs which can cause variations in the $R / Q$ values. Additionally, the mode in question is close to the beam pipe cutoff frequency and is strongly affected by the evanescent coupling of the fields in the cavity out the beam pipe aperture. The beam pipe attached to each cavity is different and can also lead to changes in the $R / Q$. Both of the above effects, while not definitive, can cause the observed differences in $R / Q$.

Using growth rates versus rf cavity temperature results it is possible to optimize operating temperatures of the main rf cavities. Since temperatures affect the transverse impedances as well as longitudinal impedances, mapping growth rates in horizontal and vertical planes is necessary for a full understanding of the trade-off. Results in all three planes have to be compared with the damping rates

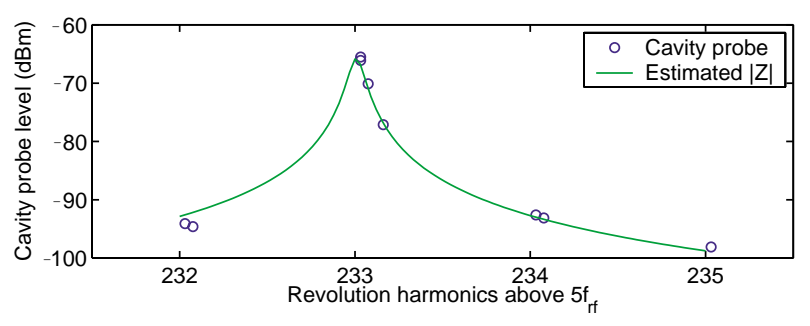

FIG. 5. (Color) Cavity 1 probe signal measured on the spectrum analyzer and the magnitude of the $2.853 \mathrm{GHz}$ impedance. 
TABLE II. Extracted impedance parameters for ALS main rf cavities compared to results of numerical computation using URMEL-T and bench measurements of a spare cavity.

\begin{tabular}{ccccc}
\hline \hline Cavity & 1 & 2 & URMEL & Spare \\
\hline$F_{r}(\mathrm{GHz})$ & 2.8532 & 2.8532 & 2.8081 & 2.85375 \\
$T_{0}\left({ }^{\circ} \mathrm{C}\right)$ & $38.61 \pm 0.07$ & $41.32 \pm 0.04$ & $\ldots$ & $\ldots$ \\
$R_{s}(\mathrm{k} \Omega)$ & $55 \pm 2$ & $97 \pm 3$ & 379 & $\ldots$ \\
$Q\left(\times 10^{3}\right)$ & $21 \pm 2$ & $24 \pm 2$ & 80.9 & 4 \\
$R / Q(\Omega)$ & $2.6 \pm 0.2$ & $4.0 \pm 0.3$ & 4.7 & $\ldots$ \\
\hline \hline
\end{tabular}

generated by the feedback systems in order to select the operating temperatures.

Since transverse growth rates have not been characterized, we have defined for each cavity a range of temperatures where longitudinal growth rates do not change significantly. As seen in Fig. 3 there are two such ranges for cavity 2: $32{ }^{\circ} \mathrm{C}-36{ }^{\circ} \mathrm{C}$ and $45^{\circ} \mathrm{C}-48{ }^{\circ} \mathrm{C}$. The lower range of temperatures is not a good choice for machine operation due to closeness to mode 205, so the higher temperature range was selected. Within this range the temperatures have additionally been optimized empirically based on the performance of the transverse feedback systems. Based on these optimizations cavity 1 was set to $46.5^{\circ} \mathrm{C}$ and cavity 2 to $45.5^{\circ} \mathrm{C}$ for nominal ALS operation.

At the selected temperatures we can estimate the relative contribution of the four different resonances driving mode 233. The growth rate can be expressed as follows:

$$
\begin{aligned}
\frac{1}{\tau_{233}}= & \operatorname{Re}\left(\Lambda_{233}\right) \\
= & \frac{\pi \alpha e f_{\mathrm{rf}}^{2} I_{0}}{E_{0} h \omega_{s}} \operatorname{Re}\left(Z_{1}^{\| \text {eff } 2.3}+Z_{1}^{\| \text {eff } 2.8}\right. \\
& \left.\quad+Z_{2}^{\| \operatorname{leff} 2.3}+Z_{2}^{\| \text {eff } 2.8}\right)-d_{r} \\
= & \frac{1}{\tau_{1}^{2.3}}+\frac{1}{\tau_{1}^{2.8}}+\frac{1}{\tau_{2}^{2.3}}+\frac{1}{\tau_{2}^{2.8}}-d_{r}
\end{aligned}
$$

where $\tau_{233}$ is the growth time of EFEM 233, $\tau_{1}^{2.3}$ and $\tau_{2}^{2.3}$ are the contributions to the growth time from the $2.353 \mathrm{GHz} \mathrm{HOM}$ in cavities 1 and 2, respectively. Similarly, $\tau_{1}^{2.8}$ and $\tau_{2}^{2.8}$ are the contributions from the $2.853 \mathrm{GHz}$ resonances in the main rf cavities.

Using extracted fit data we find:

$$
\begin{aligned}
\frac{1}{\tau_{1}^{2.8}} & =0.06 \mathrm{~ms}^{-1}, \\
\frac{1}{\tau_{2}^{2.8}} & =0.13 \mathrm{~ms}^{-1}, \\
\frac{1}{\tau_{1}^{2.3}}+\frac{1}{\tau_{2}^{2.3}} & =0.37 \mathrm{~ms}^{-1}
\end{aligned}
$$

As is evident from these numbers at the optimized cavity temperatures the contribution to the growth rate of the $2.853 \mathrm{GHz}$ impedance is $1 / 2$ of the effect of the $2.353 \mathrm{GHz}$ impedance.

\section{B. BESSY-II measurements}

These measurements were aimed at quantifying longitudinal impedances at BESSY-II [21]. The machine was filled with 350 uniformly filled consecutive bunches out of 400 to a current of $165 \mathrm{~mA}$. A series of 15 transient grow/damp experiments was conducted over a period of $10 \mathrm{~min}$. During the measurements the machine configuration remained unchanged except for the current decaying to $154 \mathrm{~mA}$. There are three unstable EFEMs seen in the data: 281, 396, and 397. According to Eq. (2), complex longitudinal impedances can be extracted from the measured growth rates and oscillation frequencies. However, these measured values are offset by $\Lambda_{0}$. Since the eigenvalue shift is small relative to the nominal synchrotron frequency $\omega_{s}$, errors in measurement of $\omega_{s}$ cause large systematic errors in the imaginary part of the extracted impedance. This difficulty can be avoided if we consider the derivative

$$
\begin{aligned}
\frac{\partial \operatorname{Im}\left(\Lambda_{l}\right)}{\partial I_{0}} & =\frac{\partial \operatorname{Im}\left(\lambda_{l}\right)}{\partial I_{0}} \\
& =\frac{\pi \alpha e f_{\mathrm{rf}}^{2}}{E_{0} h \omega_{s}} \operatorname{Im}\left[Z^{\| \text {eff }}\left(l \omega_{0}+\omega_{s}\right)\right] .
\end{aligned}
$$

A least-squares fit to the oscillation frequency component returns two coefficients: slope and offset. The slope will be used to extract the imaginary part of the impedance according to Eq. (12). The offset part corresponds to the nominal synchrotron frequency. In Fig. 6 oscillation frequencies and least-squares fits are plotted for the three unstable modes. The data for mode 281 indicate a very small imaginary part of the impedance (zero slope). At the same time modes 396 and 397 have significant imaginary impedances of opposite signs. Table III shows coefficients of the linear fits. All three fits agree on the estimate of $\omega_{s}$. Using the slope information to compute the imaginary part of the impedance and the growth rates for the real parts we get

$$
\begin{aligned}
& Z_{281}^{\| \text {eff }}=(63.2 \pm 8.1)+(0 \pm 94) j \mathrm{k} \Omega, \\
& Z_{396}^{\| \text {eff }}=(59.0 \pm 3.3)+(1115 \pm 53) j \mathrm{k} \Omega, \\
& Z_{397}^{\| \text {leff }}=(59.6 \pm 3.7)-(726 \pm 36) j \mathrm{k} \Omega .
\end{aligned}
$$

In BESSY-II there are four third-harmonic rf cavities that are designed to improve beam lifetime. During the above measurements, center frequencies of all four cavities were tuned between $3 \omega_{\mathrm{rf}}-4 \omega_{0}$ and $3 \omega_{\mathrm{rf}}-3 \omega_{0}$ (parked). Our impedance measurement for modes 396 and 397 correlates well with the impedance of the parked cavities. Since for both modes the impedance is sampled far from the resonance, the imaginary component is much larger than the real one. The larger imaginary part seen at mode 396 indicates that some of the cavities are parked closer to the fourth revolution harmonic below $3 f_{\mathrm{rf}}$. Knowing the original frequencies of these impedances allows us to compute the unaliased longitudinal impedance, 

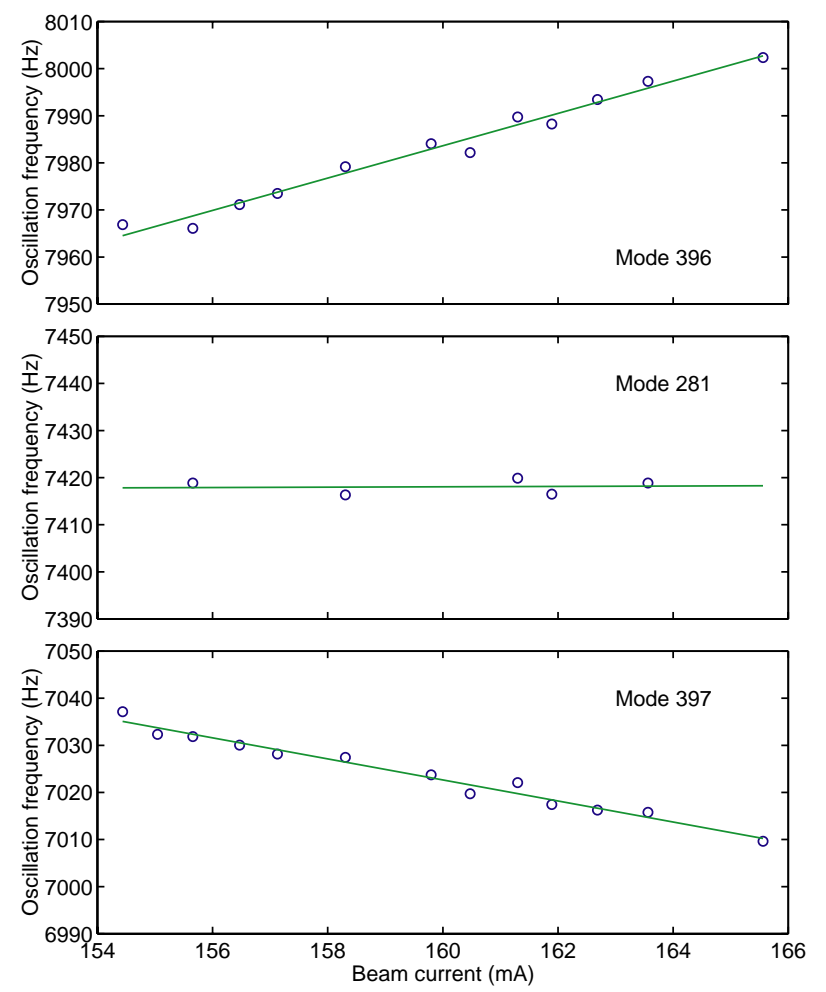

FIG. 6. (Color) Measurements of open-loop oscillation frequencies of three unstable modes and least-squares linear fits to the data. The data for mode 396 show positive slope of frequency change with beam current indicating positive imaginary part of the impedance according to Eq. (12), for mode 281-nearly zero imaginary part - while mode 397 shows negative imaginary impedance (negative slope).

$$
\begin{aligned}
& Z_{396}^{\|}=(19.7 \pm 1.1)+(373 \pm 18) j \mathrm{k} \Omega, \\
& Z_{397}^{\|}=(19.9 \pm 1.2)-(243 \pm 12) j \mathrm{k} \Omega .
\end{aligned}
$$

As described in Sec. III, we can estimate the impedance by analyzing the synchronous phase transient. In this case we estimate the impedance sampled at the revolution harmonics rather than at their upper synchrotron sidebands. However, for the high- $Q$ resonance parked between two revolution harmonics there is little change over the $\omega_{s}$ interval. In Fig. 7 synchronous phase transient in BESSY-II is presented. The top graph shows bunch-by-bunch currents with 350 consecutive buckets filled nearly equally. The periodic excitation of the impedance from the fill with gap generates oscillatory behavior of the synchronous

TABLE III. Coefficients of the linear fits to the oscillation frequencies versus beam current.

\begin{tabular}{ccc}
\hline \hline Mode & Slope $(\mathrm{Hz} / \mathrm{mA})$ & Offset $(\mathrm{Hz})$ \\
\hline 281 & $0 \pm 0.3$ & $7411 \pm 46$ \\
396 & $3.43 \pm 0.16$ & $7434 \pm 26$ \\
397 & $-2.24 \pm 0.11$ & $7380 \pm 18$ \\
\hline \hline
\end{tabular}

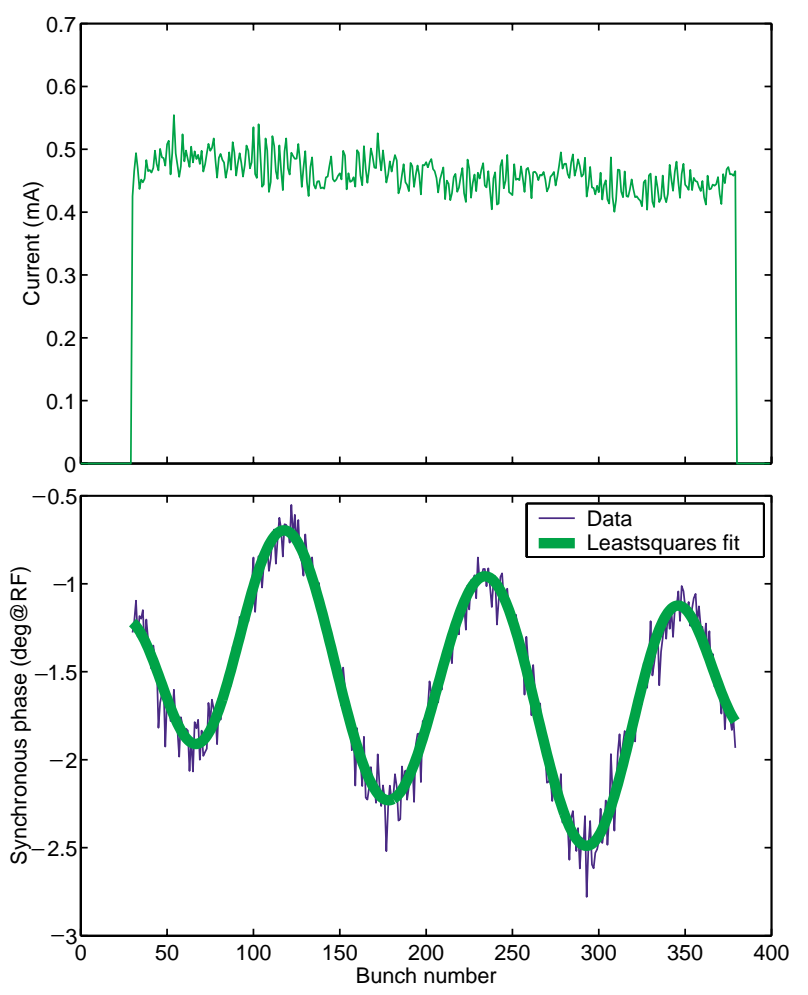

FIG. 7. (Color) Bunch-by-bunch currents (top) and synchronous phases (bottom) extracted from BESSY-II data set.

phases shown in the bottom plot. Solving Eq. (10) in the least-squares sense we obtain aliased impedances. A least-squares estimate of the synchronous phases is also shown in Fig. 7 for comparison with experimental data. Using the 15 BESSY transient measurements described above we get the following values:

$$
\begin{aligned}
& Z_{396}^{\dagger}=(35 \pm 22)+(344 \pm 14) j \mathrm{k} \Omega \\
& Z_{397}^{\dagger}=(22 \pm 6)-(233 \pm 15) j \mathrm{k} \Omega
\end{aligned}
$$

These values show reasonable agreement with the results obtained from the growth rates and tune shifts. The two methods of measuring the impedance can be used together in order to determine unaliased frequencies. This is possible due to the fact that during aliasing into $Z^{\text {lleff }}$ the impedance is scaled by resonant frequency, while in $Z^{\dagger}$ it is unscaled. Thus, from the ratio of $Z^{\text {lleff }}$ to $Z^{\dagger}$ we can estimate the frequency of the physical impedance. From Eq. (3) we have (assuming that one physical impedance dominates the aliased function)

$$
\begin{aligned}
\left|Z_{396}^{\| \text {eff }}\right| & =\frac{(p N+396) \omega_{0}}{N \omega_{0}}\left|Z_{396}^{\dagger}\right|, \\
p_{\exp } & =\frac{\left|Z_{396}^{\| \operatorname{leff}}\right|}{\left|Z_{396}^{\dagger}\right|}-\frac{396 \omega_{0}}{400 \omega_{0}}=2.2 .
\end{aligned}
$$

Since $p$ in Eq. (13) is an integer by definition, the comparison above indicates that the physical impedance is at 
$2 \omega_{\text {rf }}+396 \omega_{0}=3 \omega_{\text {rf }}-4 \omega_{0}$. This conclusion agrees perfectly with the expected position of the parked thirdharmonic cavities.

\section{SUMMARY}

We demonstrated two new methods for measuring the impedance of accelerator components using transient diagnostic capabilities of the digital signal processor based longitudinal feedback systems. The essential feature of these methods is the use of the data sets of bunch motion coordinates sampled over long time intervals. In one approach impedance is computed via measurements of complex eigenvalues of the unstable coupled-bunch modes. The second method extracts the impedance from the relationship between bunch-by-bunch currents and steady-state synchronous phases. The methods extend the capabilities of laboratory bench measurements by quantifying the physical impedances as installed in the accelerator. Dependence of the impedances on operating conditions such as temperature or tuner position can be extracted and used to select optimal working points. By comparing information obtained from growth transients with the analysis of the synchronous phase transients for uneven fills it is possible to determine the spectral position of the driving impedance.

There are certain limitations on the applicability of these methods. Transient measurement depends on the beam being unstable for it relies on growing modes of oscillation to quantify the impedances. Measurement of stable modes is possible via external excitation, but is relatively noisy due to the slow transients involved. Synchronous phase transients can be reliably observed only for large impedances. In addition, only a few revolution harmonics are usually excited by the Fourier components of the bunch current distribution. Both techniques sample the impedances at the spacing of the rotation frequency. Consequently, for the measurement of the narrow band resonances a controlled method to shift the resonator center frequency is needed. For the cases when only one of the two techniques is applicable, additional information is needed to determine unaliased spectral locations.

\section{ACKNOWLEDGMENTS}

The authors would like to thank Jorn Jacob of ESRF and Greg Stover of LBNL for help in setting up and conducting the ALS measurements. We also thank Shaukat Khan and Tom Knuth for setting up and taking BESSY-II transient measurements. We are very grateful to Albert Hofmann for his insightful comments on this work. This work was supported by DOE Contract No. DE-AC03-76SF00515.
[1] L. Palumbo and V.G. Vaccaro, in Frontiers of Particle Beams: Observation, Diagnosis and Correction (SpringerVerlag, Berlin, 1989), pp. 312-354.

[2] J.N. Corlett and J.M. Byrd, in Proceedings of the 1993 IEEE Particle Accelerator Conference, Washington, DC (IEEE, Piscataway, NJ, 1994), pp. 3408-3410.

[3] M. A. Allen, J. M. Paterson, J.R. Rees, and P. B. Wilson, IEEE Trans. Nucl. Sci. 22, 1838 (1975).

[4] B. Podobedov and R. Siemann, Phys. Rev. ST Accel. Beams 1, 072801 (1998).

[5] B. W. Zotter and S. A. Kheifets, Impedances and Wakes in High-Energy Particle Accelerators (World Scientific, Singapore, 1998).

[6] W. Anders, P. Kuske, and T. Westphal, in Proceedings of the 1992 Conference on High Energy Accelerators (HEACC 92), Hamburg, Germany (World Scientific, Singapore, 1993), pp. 1121-1123.

[7] D. Möhl and A. Sessler, in Proceedings of the 8th International Conference on High Energy Accelerators, Geneva, 1971 (CERN, Geneva, 1971), pp. 334-337.

[8] A. Hofmann and B. Zotter, IEEE Trans. Nucl. Sci. 24, 1487 (1977).

[9] A. Temnykh, D. Hartill, S. Belomestnykh, and R. Kaplan, Cornell University Technical Report No. CBN 97-5, 1997 (unpublished).

[10] J.L. Laclare, in Proceedings of the Conference on High Energy Accelerators (Birkhauser, Geneva, 1980), pp. 526539.

[11] A. W. Chao, Physics of Collective Beam Instabilities in High Energy Accelerators (Wiley, New York, 1993).

[12] S. Prabhakar, Ph.D. thesis, Stanford University [SLAC Report No. SLAC-R-554, 2000].

[13] J. Fox et al., in Proceedings of the 1999 Particle Accelerator Conference, New York (IEEE, Piscataway, NJ, 1999), pp. 636-640.

[14] S. Prabhakar, J. D. Fox, D. Teytelman, and A. Young, Phys. Rev. ST Accel. Beams 2, 084401 (1999).

[15] S. Prabhakar et al., in Proceedings of the Sixth European Particle Accelerator Conference, Stockholm, 1998 (Institute of Physics, Bristol, UK, 1998), pp. 996-998.

[16] S. Prabhakar et al., Part. Accel. 57, 175 (1997).

[17] J. M. Byrd et al., in Proceedings of the International Workshop on Collective Effects and Impedance for B Factories, Tsukuba, Japan, 1995 (KEK, Tsukuba, 1996), pp. 527545.

[18] M. Svandrlik et al., in Proceedings of the 1995 Particle Accelerator Conference and International Conference on High-Energy Accelerators, Dallas, Texas (IEEE, Piscataway, NJ, 1996), pp. 2762-2764.

[19] M. Kwon et al., in Proceedings of the 1999 Particle Accelerator Conference, New York (Ref. [13]), pp. 899-901.

[20] J. M. Byrd and J. Corlett, Part. Accel. 51, 29 (1995).

[21] R. J. Bakker, in Proceedings of the 1999 Particle Accelerator Conference, New York (Ref. [13]), pp. 197-199. 